\title{
Clinical outcomes of hysterectomy for benign diseases in the female genital tract: 6 years' experience in a single institute
}

\author{
Hyo-Shin Kim ${ }^{1}$, Yu-Jin Koo ${ }^{2}$, Dae-Hyung Lee ${ }^{2}$ \\ ${ }^{1}$ Department of Obstetrics and Gynecology, Yeungnam University Hospital, Daegu, Korea \\ ${ }^{2}$ Department of Obstetrics and Gynecology, Yeungnam University College of Medicine, Daegu, Korea
}

Received: March 17, 2020

Revised: April 7, 2020

Accepted: April 14, 2020

Corresponding author:

Yu-Jin Koo

Department of Obstetrics and

Gynecology, Yeungnam University

College of Medicine, 170

Hyeonchung-ro, Nam-gu, Daegu

42415 , Korea

Tel: +82-53-620-3433

Fax: +82-53-654-0676

E-mail:yujinkoo@yu.ac.kr

\begin{abstract}
Background: Hysterectomy is one of the major gynecologic surgeries. Historically, several surgical procedures have been used for hysterectomy. The present study aims to evaluate the surgical trends and clinical outcomes of hysterectomy performed for benign diseases at the Yeungnam University Hospital.

Methods: We retrospectively reviewed patients who underwent a hysterectomy for benign diseases from 2013 to 2018. Data included the patients' demographic characteristics, surgical indications, hysterectomy procedures, postoperative pathologies, and perioperative outcomes.

Results: A total of 809 patients were included. The three major indications for hysterectomy were uterine leiomyoma, pelvic organ prolapse, and adenomyosis. The most common procedure was total laparoscopic hysterectomy (TLH, 45.2\%), followed by open hysterectomy (32.6\%). During the study period, the rate of open hysterectomy was nearly constant (29.4\%-38.1\%). The mean operative time was the shortest in the single-port laparoscopic assisted vaginal hysterectomy (LAVH, 89.5 minutes), followed by vaginal hysterectomy (VH, 96.8 minutes) and TLH (105 minutes). The mean decrease in postoperative hemoglobin level was minimum in single-port LAVH $(1.8 \mathrm{~g} / \mathrm{dL})$ and $\mathrm{VH}(1.8 \mathrm{~g} / \mathrm{dL})$. Conversion to open surgery or multi-port surgery occurred in five cases $(0.6 \%)$. Surgical complications including wound dehiscence, organ injuries, and conditions requiring reoperation were observed in 52 cases (6.4\%).

Conclusion: Minimally invasive approach was used for most hysterectomies for benign diseases, but the rate of open hysterectomy has mostly remained constant. Single-port LAVH and VH showed the most tolerable outcomes in terms of operative time and postoperative drop in hemoglobin level in selected cases.
\end{abstract}

Keywords: Female genital disease; Gynecological surgical procedures; Hysterectomy; Minimally invasive surgical procedures

\section{Introduction}

Hysterectomy is one of the major gynecologic surgeries. In Korea, more than 40,000 cases of hysterectomy are performed each year and it was ranked 6th among all main surgeries in Korea in 2017 according to the data by Korean National Health Insurance Service [1]. There are three major types of hysterectomy procedures: open abdominal surgery, vaginal surgery, and endoscopic surgery.

Copyright (C) 2020 Yeungnam University College of Medicine

This is an Open Access article distributed under the terms of the Creative Commons Attribution Non-Commercial License (http://creativecommons.org/licenses/by-nc/4.0/) which permits unrestricted non-commercial use, distribution, and reproduction in any medium, provided the original work is properly cited. 
With the growing desire for minimally invasive surgery (MIS), endoscopic approaches, such as total laparoscopic hysterectomy (TLH), laparoscopy-assisted vaginal hysterectomy (LAVH), and robot-assisted laparoscopic hysterectomy (RALH), have become prevalent in the field of hysterectomy. In particular, RALH is a new minimally invasive technique that has some theoretical advantages including improved visualization through three-dimensional imaging, greater precision, and more accurate control of instrumentation in addition to improved ergonomics for the surgeons [2].

In June 2017, the American College of Obstetricians and Gynecologists (ACOG) published a committee opinion, presenting vaginal hysterectomy $(\mathrm{VH})$ as an approach of choice and recommending laparoscopic hysterectomy (including LAVH) as an alternative when a VH is not indicated or feasible [3]. The choice of surgical approach for gynecologic malignancy might be limited; however, for benign diseases, various surgical approaches can be selected depending on the individual case. This study aimed to review the trends in surgical procedures of hysterectomy for benign diseases and to evaluate the surgical outcomes of each procedure performed in our center over a 6-year period.

\section{Materials and methods}

We retrospectively enrolled women who underwent hysterectomy for a benign genital tract disease during a 6-year period between January 1, 2013, and October 31, 2018, at the Department of Obstetrics and Gynecology of Yeungnam University Hospital, Daegu, Korea. The study was approved by the Institutional Review Board (IRB) at Yeungnam University Hospital (IRB No: 2019-12-050). Medical records of the participants, including outpatient records, imaging and laboratory reports, surgical records, postoperative progress notes, and pathologic reports were reviewed. Factors examined included patient's clinical characteristics, symptoms, preoperative diagnosis, surgical modes, concurrent procedures with hysterectomy, postoperative diagnosis, and surgical outcomes including complications.

Laparoscopic hysterectomy is typically classified into two categories, namely TLH and LAVH, depending on the extent of the procedure performed laparoscopically or vaginally. In TLH, the entire procedure, except the removal of the uterus, is performed laparoscopically. LAVH differs from TLH in that the procedures, including ligation of the uterine vessels, colpotomy, and suturing of the vaginal vault, are done using the vaginal approach. In our hospital, TLH has been performed only by multi-port approach, and meanwhile, LAVH has been performed through either a multi-port or a single-port approach, since a single-port hysterec- tomy was first introduced in 2018. In a laparoscopic multi-port surgery, four holes were made as follows: an umbilical hole for laparoscope and a suprapubic and two lateral holes for instruments. In a single-port surgery, a surgeon made an incision of 2 $\mathrm{cm}$ at the umbilicus and used a commercial multichannel port, Glove port (Nelis, Bucheon, Korea) to insert a rigid 5-mm 30 laparoscopic scope and two rigid 5-mm instruments. In our hospital, the DaVinci Si surgical system was introduced on September 6, 2013. RALH was also performed by either TLH or LAVH and by either a multi-port or a single-port approach. The hysterectomy of this present study was performed by a total of seven operators, and their surgical experience in hysterectomy varied from 3 years to over 20 years. RALH was performed by three operators and single-port LAVH was performed by a single operator.

The operative time was measured from the time of skin incision to the time of skin closure completion. In the case of RALH, the docking time from the port placement to the docking of the robot was included in the operative time. The postoperative hemoglobin $(\mathrm{Hb})$ drop was calculated by subtracting the $\mathrm{Hb}$ level at the first postoperative day from the preoperative $\mathrm{Hb}$ level. For patients who received intra- or postoperative transfusion, the lowest $\mathrm{Hb}$ level measured before transfusion was used as postoperative $\mathrm{Hb}$ level. The patients who received transfusion without the $\mathrm{Hb}$ test were excluded from the calculation of the $\mathrm{Hb}$ drop. Only the cases with wound defect requiring resuturing were reported as complications of wound dehiscence.

Hospital stay was measured from the day after surgery to the day of discharge. Before 2016, patients who underwent laparoscopic hysterectomy were generally discharged on the 4-5th postoperative day, but after 2017, they were discharged on the 2nd postoperative day in line with on our hospital policy. For open surgery with pfannenstiel skin incision, patients are usually discharged on the 4-6th postoperative day and on the 5-7th postoperative day in the case of low-midline incision. Patients who have undergone $\mathrm{VH}$ are discharged on the $4-5$ th postoperative day.

\section{Results}

A total of eight hundred and nine patients were included in the analysis. The mean age of the patients was 53.4 years and the mean body mass index (BMI) was $24.6 \mathrm{~kg} / \mathrm{m}^{2}$ (Table 1). Patients with previous abdominal surgery accounted for $37.6 \%$. The three most common symptoms were abnormal uterine bleeding (28.7\%), protruding prolapse of pelvic organs (17.7\%), and abdominal pain or discomfort (16.1\%). The common indications for hysterectomy, namely preoperative diagnosis, were uterine leiomyoma (47.5\%), pelvic organ prolapse (17.7\%), and adeno- 
Table 1. Clinical characteristics of the patients and surgical indications for hysterectomy $(n=809)$

\begin{tabular}{|c|c|}
\hline Clinical characteristic & Value \\
\hline Age (yr) & $53.4(30-88)$ \\
\hline Gravidity (no. of times) & $3.8(0-15)$ \\
\hline Parity (no. of times) & $2.2(0-8)$ \\
\hline Body mass index $\left(\mathrm{kg} / \mathrm{m}^{2}\right)$ & $24.6(16.4-40.4)$ \\
\hline History of abdominopelvic surgery & $304(37.6)$ \\
\hline \multicolumn{2}{|l|}{ Chief complaints } \\
\hline No symptom & $200(24.7)$ \\
\hline Abnormal uterine bleeding & $232(28.7)$ \\
\hline Protruding prolapse of pelvic organs & $143(17.7)$ \\
\hline Abdominal pain or discomfort & $130(16.1)$ \\
\hline Dysmenorrhea & $47(5.8)$ \\
\hline Palpable pelvic mass & $21(2.6)$ \\
\hline Urinary symptoms & $19(2.3)$ \\
\hline Vaginal discharge & $9(1.1)$ \\
\hline Others & $8(1.0)$ \\
\hline \multicolumn{2}{|l|}{ Surgical indications ${ }^{\text {a) }}$} \\
\hline Uterine leiomyoma & $384(47.5)$ \\
\hline Pelvic organ prolapse & $143(17.7)$ \\
\hline Adenomyosis or adenomyoma & $108(13.3)$ \\
\hline Cervical intraepithelial neoplasia & $71(8.8)$ \\
\hline Adnexal lesion & $62(7.7)$ \\
\hline Endometrial hyperplasia or polyp & $20(2.5)$ \\
\hline Postpartum uterine atony & $7(0.9)$ \\
\hline \multicolumn{2}{|l|}{ Others } \\
\hline Hematometra or hydrometra & $3(0.4)$ \\
\hline Placenta accreta, increta & $3(0.4)$ \\
\hline Abnormal uterine bleeding & $2(0.2)$ \\
\hline Pelvic actinomycosis & $2(0.2)$ \\
\hline Pelvic endometriosis & $2(0.2)$ \\
\hline Hydatidiform mole & $1(0.1)$ \\
\hline Tubo-ovarian abscess & $1(0.1)$ \\
\hline
\end{tabular}

Values are presented as mean (range) or number (\%).

${ }^{\text {a) }} \mathrm{A}$ main diagnosis that was assumed clinically before hysterectomy was described.

myosis or adenomyoma (13.3\%).

The most frequently performed procedure was TLH (45.2\%), followed by total abdominal hysterectomy (TAH, 32.6\%), VH (16.2\%), LAVH (4.9\%), robotic TLH (0.6\%), and robotic LAVH (0.4\%) (Table 2$)$. In three cases $(0.4 \%)$, subtotal hysterectomy was done unexpectedly due to technical challenges of the total hysterectomy. Among 414 cases of laparoscopic or robotic surgery, 15 cases $(3.6 \%)$ were done using the single-port approach (11 cases of LAVH, two cases of robotic TLH, and two cases of robotic LAVH). The pathologic result confirmed the postoperative diagnosis as uterine leiomyoma or adenomyosis in more than half of the patients (505 cases, 62.4\%).
Table 2. Surgical procedures for hysterectomy and postoperative diagnosis $(n=809)$

\begin{tabular}{|c|c|}
\hline Surgical procedure & No. $(\%)$ \\
\hline \multicolumn{2}{|l|}{ Hysterectomy procedure } \\
\hline $\mathrm{TAH}$ & $264(32.6)$ \\
\hline TLH & $366(45.2)$ \\
\hline LAVH & $40(4.9)$ \\
\hline \multicolumn{2}{|l|}{ Robot-assisted } \\
\hline Robotic TLH & $5(0.6)$ \\
\hline Robotic LAVH & $3(0.4)$ \\
\hline Vaginal hysterectomy & $131(16.2)$ \\
\hline \multicolumn{2}{|c|}{ Port number for laparoscopic or robotic surgery } \\
\hline Single-port & $15 / 414^{\mathrm{a})}(3.6)$ \\
\hline Multi-port & $399 / 414^{\text {a) }}(96.4)$ \\
\hline \multicolumn{2}{|c|}{ Procedures combined with hysterectomy } \\
\hline Bilateral salpingoophorectomy & $259(32)$ \\
\hline Colporrhaphy & $138(17.1)$ \\
\hline Unilateral salpingoophorectomy & $71(8.8)$ \\
\hline Ovarian cystectomy & $16(2)$ \\
\hline Others & $20(2.5)$ \\
\hline \multicolumn{2}{|l|}{ Postoperative diagnosis } \\
\hline Uterine leiomyoma & $377(46.6)$ \\
\hline Prolapsed pelvic organs & $143(17.7)$ \\
\hline Adenomyosis or adenomyoma & $128(15.8)$ \\
\hline Cervical intraepithelial neoplasia & $72(8.9)$ \\
\hline Endometrial or endocervical polyp & $7(0.9)$ \\
\hline Uterine atony & $7(0.9)$ \\
\hline Pelvic inflammatory disease & $4(0.5)$ \\
\hline Endometrial hyperplasia & $3(0.4)$ \\
\hline Placenta increta, accreta & $3(0.4)$ \\
\hline Adnexal lesion & $60(7.4)$ \\
\hline Unremarkable $^{\text {b) }}$ & $2(0.2)$ \\
\hline \multicolumn{2}{|l|}{ Others } \\
\hline Adenomatoid tumor & $1(0.1)$ \\
\hline STUMP & $1(0.1)$ \\
\hline Hydatidiform mole & $1(0.1)$ \\
\hline
\end{tabular}

TAH, total abdominal hysterectomy; $T L H$, total laparoscopic hysterectomy; LAVH, laparoscopy-assisted vaginal hysterectomy; STUMP, smooth muscle tumor of uncertain malignant potential.

${ }^{a}$ Denominators are the number of the total case of laparoscopic and

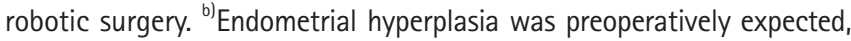
but the pathologic diagnosis was normal.

Mean operative time was shortest in single-port LAVH (89.5 minutes), followed by VH (96.8 minutes) and TLH (105 minutes) (Table 3). Mean decrease in postoperative $\mathrm{Hb}$ level was also minimal in single-port LAVH $(1.8 \mathrm{~g} / \mathrm{dL})$ and VH $(1.8 \mathrm{~g} / \mathrm{dL})$. The average number of hospital days was 2.2 days (range, 2-3 days) in single-port LAVH, 3.5 days (range, 2-13 days) in TLH, 5.4 days (range, 2-18 days) in multi-port LAVH, 6.3 days (range, 2-25 days) in RALH, 6.3 days (range, 2-38 days) in $\mathrm{VH}$, and 7.4 
Table 3. Operative time and postoperative hemoglobin drop according to the surgical procedures

\begin{tabular}{lc}
\hline Surgical outcome & Mean (range) \\
\hline Operative time (min) & $137.1(65-290)$ \\
TAH & \\
LAVH & $89.5(70-125)$ \\
$\quad$ Single-port & $181.2(80-495)$ \\
Multi-port & $105.0(60-250)$ \\
TLH & \\
Robot & $133.8(75-240)$ \\
Single-port & $273.8(215-325)$ \\
Multi-port & $96.8(60-170)$ \\
Vaginal hysterectomy & \\
Hemoglobin drop after surgery (g/dL) & $1.9(0-8.5)$ \\
TAH & \\
LAVH & $1.8(0-3.6)$ \\
Single-port & $2.6(0.9-5.5)$ \\
Multi-port & $1.9(0-5.9)$ \\
TLH & \\
Robot & $3.1(1.9-4.9)$ \\
Single-port & $2.4(1.6-4.1)$ \\
Multi-port & $1.8(0-6.1)$ \\
Vaginal hysterectomy & \\
\hline
\end{tabular}

TAH, total abdominal hysterectomy; TLH, total laparoscopic hysterectomy; LAVH, laparoscopy-assisted vaginal hysterectomy.

days (range, 2-59 days) in TAH. In the entire group, the mean length of hospital stay was 5.3 days (Table 4).

Endoscopic surgery was converted to open surgery in four cases $(0.5 \%)$, all from TLH to open surgery. Two of the converted cases were due to severe pelvic adhesion, and two other cases due to poor surgical exposure caused by huge uterine mass. Single-port surgery was converted to multi-port surgery in a case $(0.1 \%)$, which was a conversion from single-port RALH to multi-port TLH due to severe pelvic adhesion. Among a total of five patients who experienced conversion, four were overweighted (BMI, 23$\left.24.9 \mathrm{~kg} / \mathrm{m}^{2}\right)$ or obese $\left(\right.$ BMI, $\left.\geq 25 \mathrm{~kg} / \mathrm{m}^{2}\right)$.

Transfusion due to surgical bleeding was done in $7.5 \%$ (Table 4). Intra- and postoperative complications were reported in 52 cases, including wound dehiscence, organ injury, fistula, wound hematoma or infection, bowel obstruction, incisional hernia, and thromboembolism. Twenty-one patients (2.6\%) experienced reoperation due to complications: hematoma removal and bleeding control in six, wound dehiscence repair in five, ureter injury repair in four, fistula repair in three, incisional hernia repair in two, and bowel injury repair in one. The most common complication was wound dehiscence $(2 \%)$, and a total of four cases with vaginal stump dehiscence were reported in TLH. Urinary injury and bowel injury occurred in $1.4 \%$ and $0.4 \%$, respectively. Of the five
Table 4. Perioperative outcomes including surgical complications $(n=809)$

\begin{tabular}{lc}
\hline Perioperative outcome & Value \\
\hline Hospital stay (day) & $5.3(2-59)$ \\
Conversion to open surgery & $4(0.5)$ \\
Conversion from single-port to multi-port surgery & $1(0.1)$ \\
Transfusion due to surgical bleeding & $61(7.5)$ \\
Intra- and postoperative complications & $52(6.4)$ \\
Reoperation & \\
Hematoma removal and bleeding control & $6(0.7)$ \\
Wound dehiscence repair ${ }^{a}$ & $5(0.6)$ \\
Ureter injury repair & $4(0.5)$ \\
Fistula repair & $3(0.4)$ \\
Incisional hernia repair & $2(0.2)$ \\
Bowel injury repair & $1(0.1)$ \\
Wound dehiscence & \\
Low-midline skin incision site & $6 / 109^{\mathrm{c})}(5.5)$ \\
Pfannenstiel skin incision site & $5 / 153^{\mathrm{c})}(3.3)$ \\
Vaginal stump & $4 / 809^{\mathrm{c}}(0.5)$ \\
Perineum after posterior colporrhaphy & $1 / 138^{\mathrm{c})}(0.7)$ \\
Fistula & \\
Ureterovaginal & $2(0.2)$ \\
Sigmoid colovaginal & $1(0.1)$ \\
Organ injury & \\
Bladder & $5(0.6)$ \\
Ureter & $5(0.6)$ \\
Bowel & $3(0.4)$ \\
\hline & $1(0.1)$ \\
\hline
\end{tabular}

Values are presented as mean (range) or number (\%).

${ }^{a)}$ The cases includes only the cases that required resuturing in the operating room under general anesthesia. ${ }^{b}$ The cases includes only the cases that required resuturing. ${ }^{\mathrm{C}}$ Denominator refers to the number of the cases of low-midline skin incision, pfannenstiel skin incision, colpotomy, and posterior colporrhaphy, respectively.

cases of bladder injury, four were detected intraoperatively and treated through immediate primary repair. The other one was detected on 2nd postoperative day and received reoperation for surgical repair by the urology department. In the case of ureter injury, all five were detected on the days after surgery. One out of five underwent ureteral stent insertion, while the other four received ureteroneocystostomy. In the case of patients with both bladder and ureter injury, as was detected intraoperatively, immediate primary repair of bladder and ureteroneocystostomy were performed. Among three cases of bowel injury, two were detected intraoperatively, and an immediate primary repair was performed. Meanwhile, the other one was found on the 7th postoperative day and underwent a laparotomy for primary repair of rectal perforation and ileostomy. Two cases of ureterovaginal fistula occurred after multi-port LAVH and were treated through ureteral stent inser- 
tion or ureteroneocystostomy. A case of sigmoid colovaginal fistula developed after TLH and was repaired by the colorectal surgery department.

The complication rate was highest in multi-port LAVH (7/29, $24.1 \%)$, followed by RALH (1/8, 12.5\%), TAH (27/264, 10.2\%), TLH (18/366, 4.9\%), VH (4/131, 3.1\%), and single-port LAVH $(0 / 11)$. Likewise, the rate of reoperation was highest in multi-port LAVH (4/29, 13.8\%), but no case of reoperation was found in both $\mathrm{VH}$ and single-port LAVH. None of the patients died as a result of hysterectomy.

\section{Discussion}

Uterine leiomyoma is the most common indication for hysterectomy and is also the most common disease that is revealed on the postoperative pathologic result [1]. According to data by the Korean National Health Insurance Service, hysterectomy due to uterine leiomyoma was the 20th most frequent operation among surgeries due to a single disease in 2017 [1]. That was the only gynecological surgery on the list of the top 20 .

Fig. 1 shows a surgical mode for hysterectomy by year in a total of 809 patients. MIS such as TLH, LAVH, RALH, and VH has been performed approximately in two-thirds of the cases but the rate of open hysterectomy has remained almost constant (29.4\%-38.1\%) without noticeable decrease. This is probably because TAH is still preferable to MIS in some cases with huge uterine mass, severe pelvic adhesion, risk of malignancy, and patient's medical diseases. In addition, cases of cesarean hysterectomy, which cannot be replaced by MIS, were included in the cases of TAH.
A 2015 Cochrane meta-analysis reviewed randomized controlled trials and compared the clinical outcomes of different surgical approaches to hysterectomy for benign gynecological disease [4]. In the result, there were no significant differences in most of the surgical outcomes between TLH and LAVH, including hospital stay, intraoperative visceral injury, conversion to open surgery, vaginal cuff infection, transfusion, etc. The only significant difference was in operation time; LAVH (115.1 minutes) had a shorter operation time than TLH (140.4 minutes) (mean difference, -23.3 minutes; 95\% confidence interval, -10.0 to -40.6$)$. No evidence of a difference was found between single-port and multi-port laparoscopic hysterectomy for intraoperative complication, operation time, and hospital stay. However, the authors found that laparoscopic hysterectomy was associated with more urinary tract injuries than abdominal hysterectomy was. The incidence rate of urinary tract injury was $4.2 \%$ in TLH and 3.3\% in LAVH, with no statistically significant difference. In our study, urinary tract injury was the most commonly encountered organ injury. It occurred in $1.9 \%$ of TLH (7 out of 366), but in $10 \%$ ( 4 out of 40 ) of LAVH, which is relatively high. This high incidence of urinary tract injury in LAVH group seems to be related with the surgeon's operative skill rather than the mode of hysterectomy because all events in LAVH group were occured in multi-port surgery by a single surgeon. No case of urinary tract injury was found in both $\mathrm{VH}$ and TAH.

In this study, single-port LAVH and $\mathrm{VH}$ showed the best surgical outcomes in terms of operation time, postoperative $\mathrm{Hb}$ drop, and surgical complication, although the number of cases was small and selection bias maybe exist. Compared to the results from the previously reported studies regarding LAVH [4-6], our

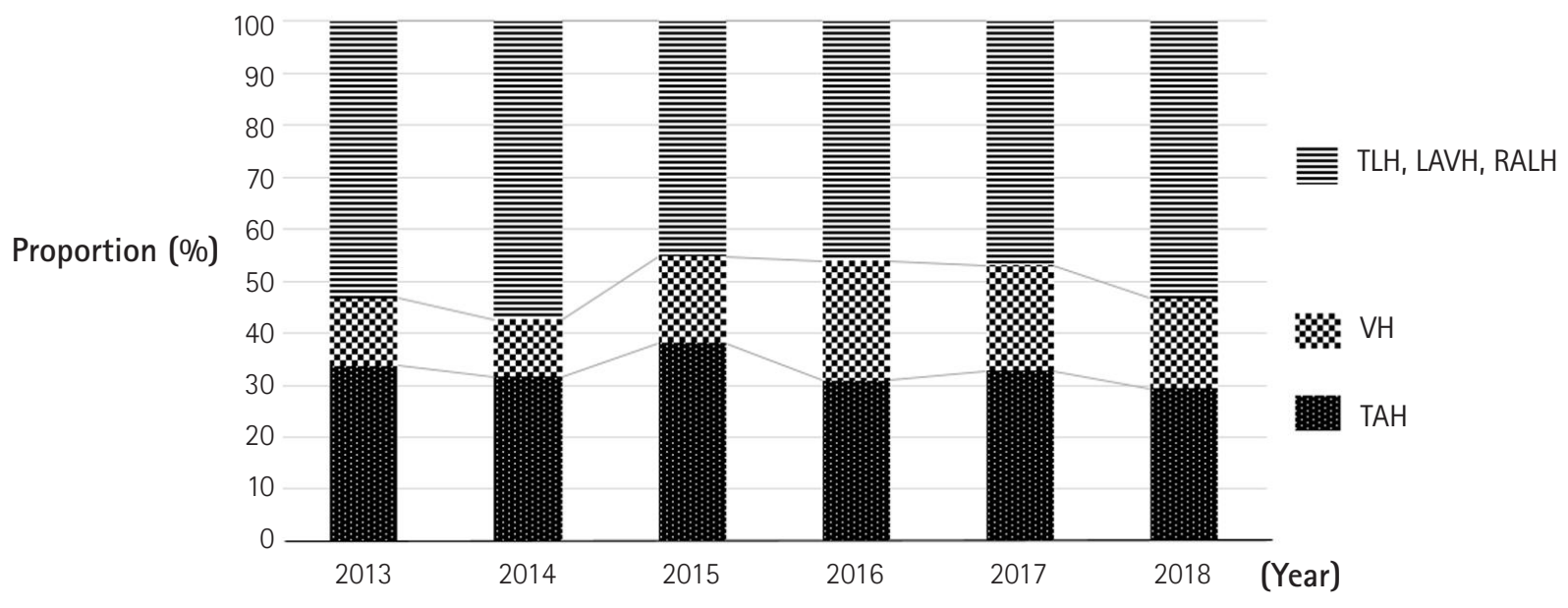

Fig. 1. Trend in procedures of hysterectomy for benign diseases during 6 years of experience. TLH, total laparoscopic hysterectomy; LAVH, laparoscopy-assisted vaginal hysterectomy; RALH, robot-assisted laparoscopic hysterectomy; VH, vaginal hysterectomy; TAH, total abdominal hysterectomy. 
data shows tolerable surgical outcomes. In a German retrospective study [5], the mean operation time and postoperative $\mathrm{Hb}$ drop were 137 minutes and $1.8 \mathrm{~g} / \mathrm{dL}$, respectively, for LAVH (the port number was not described in the study). Another study reported 91.2 minutes of the mean operation time and $1.6 \mathrm{~g} / \mathrm{dL}$ of $\mathrm{Hb}$ drop for single-port LAVH in a prospective setting [6]. The authors have suggested that both single-port TLH and single-port LAVH are feasible with similar surgical outcomes but single-port LAVH may be preferred in patients with a uterus with a large lower uterine segment.

Since single-port surgery has been introduced recently in our hospital and a surgeon had already become proficient in $\mathrm{LAVH}$, it is no wonder that single-port LAVH showed the good clinical outcomes. Moreover, a surgeon may have performed single-port LAVH or VH in highly-selected cases, such as uterus with small masses and no adhesion. In other words, if the case is well-selected, single-port $\mathrm{LAVH}$ and $\mathrm{VH}$ are safe and profitable for benign uterine disease. In the case of $\mathrm{VH}$, the surgical outcome might be undervalued in our study. We usually perform VH in patients with uterine prolapse; therefore, the good surgical outcomes might be offset by several factors such as patients' old age, medical disease, and concurrent procedures (e.g., colporrhaphy). Given the increasing global need for MIS and its possible cosmetic advantage, it is speculated that single-port LAVH has clear benefits and will be more popular. Therefore, it is necessary for clinicians to evaluate the trend in surgical approaches performed in their institute and to determine the clinical outcomes according to the different surgical procedures.

The present study has several limitations. First, it is a retrospective study. Second, the important confounding factors that can affect clinical outcomes, such as the surgeon's skill and the patient's clinical characteristics, were not analyzed according to the type of surgical approaches. Third, the number of patients in the subgroups of LAVH and RALH were too small, which precludes definite conclusions from being drawn. Future studies, with larger sample sizes and adjustments for potential confounders, are warranted to evaluate the significant differences between approaches.

In conclusion, the minimally invasive approach is used in most hysterectomies for benign diseases, but the rate of open hysterectomy has remained almost constant in our hospital. Single-port $\mathrm{LAVH}$ and VH seem to be the most feasible and safe, in terms of short operative time, minimal decrease in postoperative $\mathrm{Hb}$ level, and low incidence of complication in selected cases. Although the number of single-port surgeries was small, their remarkable supe- rior outcomes suggest that single-port LAVH is potentially one of the best options for benign uterine disease.

\section{Acknowledgments}

\section{Conflicts of interest}

No potential conflict of interest relevant to this article was reported.

\section{Author contributions}

Conceptualization: YJK; Data curation: HSK; Formal analysis: HSK, YJK; Project administration: YJK; Supervision: DHL; Writing-original draft: HSK; Writing-review \& editing: YJK.

\section{ORCID}

Hyo-Shin Kim, https://orcid.org/0000-0002-8369-417X

Yu-Jin Koo, https://orcid.org/0000-0002-5114-8000

Dae-Hyung Lee, https://orcid.org/0000-0002-0219-0317

\section{References}

1. Korea National Health Insurance Service (NHIS). The main surgery statistical yearbook for 2017 [Internet]. Wonju: NHIS; 2018 [cited 2020 Mar 10]. https://www.nhis.or.kr/menu/ boardRetriveMenuSet.xx?menuId = F3326.

2. Nevis IF, Vali B, Higgins C, Dhalla I, Urbach D, Bernardini MQ. Robot-assisted hysterectomy for endometrial and cervical cancers: a systematic review. J Robot Surg 2017;11:1-16.

3. Committee on Gynecologic Practice. Committee opinion no. 701: choosing the route of hysterectomy for benign disease. Obstet Gynecol 2017;129:e155-9.

4. Aarts JW, Nieboer TE, Johnson N, Tavender E, Garry R, Mol BW, et al. Surgical approach to hysterectomy for benign gynaecological disease. Cochrane Database Syst Rev 2015;(8): CD003677.

5. Elessawy M, Schollmeyer T, Mettler L, Jonat W, Schem C, von Hehn U, et al. The incidence of complications by hysterectomy for benign disease in correlation to an assumed preoperative score. Arch Gynecol Obstet 2015;292:127-33.

6. Song T, Kim MK, Kim ML, Jung YW, Yun BS, Seong SJ. A randomized comparison of laparoendoscopic single-site hysterectomies: total laparoscopic hysterectomy versus laparoscopically assisted vaginal hysterectomy. J Laparoendosc Adv Surg Tech A 2015;25:541-7. 